\title{
IDENTIFICATION, CHARACTERIZATION AND ANTIMICROBIAL SUSCEPTIBILITY TESTING OF ENTEROCOCCUS SPECIES ISOLATED FROM VARIOUS CLINICAL SPECIMENS
}

\author{
Amar Pandya1, M.B. Shah², Sunil Trivedi ${ }^{3}$, Suman Singh ${ }^{4}$
}

\section{HOW TO CITE THIS ARTICLE:}

Amar Pandya, M.B. Shah, Sunil Trivedi, Suman Singh."Identification, Characterization and Antimicrobial Susceptibility Testing of Enterococcus Species isolated from various Clinical Specimens". Journal of Evolution of Medical and Dental Sciences 2014; Vol. 3, Issue 02, January 13; Page: 289-294,

DOI:10.14260/jemds/2014/1823

ABSTRACT: CONTEXT: The emergence of acquired resistant to glycopeptides (vancomycin) and high level aminoglycosides in serious enterococcal infections are extremely difficult to treat and cause significant morbidity and mortality. AIMS: The aim of the present study was to characterize enterococcus species from various clinical specimens and describe their antimicrobial resistance pattern particularly to high level aminoglycosides and vancomycin. METHODS AND MATERIAL: The study was conducted at a tertiary care hospital in India. Identification of enterococci was done by miniAPI and Antibiotic susceptibility was checked according to CLSI guidelines 2011.RESULTS: Out of 62 enterococcal isolates $40 \%$ were E.faecalis followed by 32\% E.faecium and 17\% E.gallinarum. Forty four (71\%) enterococcal species were high level aminoglycoside resistant with E.faecium (90\%) being predominant followed by E.faecalis (71\%). MIC of high level gentamicin resistant strains were found to be $>1024 \mu \mathrm{g} / \mathrm{ml}$. Vancomycin resistant enterococci have been found in eight (13\%) patients with E.faecium and E.gallinarum infections. MIC of vancomycin was found to be high i.e. 32 to more than $256 \mu \mathrm{g} / \mathrm{ml}$. CONCLUSIONS: Enterococci are identified as major pathogens causing infections in hospital as well as community. Resistance to beta-lactams and high level aminoglycoside was found to be significantly high $(\mathrm{P}=<0.05)$. Such high resistance rates among isolates in present study limits the treatment options available and prompt measures should be initiated to control their spread. VRE has also been found to cause infections in community in present study and so screening for VRE in newly admitted patient who are at high risk of acquiring hospital infections is also recommended.

KEY-WORDS:Enterococci, Vancomycin resistant enterococci, High level aminoglycoside resistance.

Key Messages: Formulating an antimicrobial policy and its strict implementation and regular surveillance of enterococcal infections and their antimicrobial profile should be conducted timely and appropriate infection control measures should be initiated to control spread of drug resistant enterococci.

INTRODUCTION: Nosocomial infections caused by enterococci have been increasing in incidence during last several years to such an extent that enterococci are listed as one of most commonly isolated pathogens. ${ }^{1}$ Combination therapy with a cell wall active agent and an aminoglycoside is recommended for serious enterococcal infections 2 but increasing resistance to first line antimicrobial agents and emergence and spread of species resistant to Vancomycin and High Level Gentamicin have reduced therapeutic options to treat several enterococcal infections. ${ }^{3}$ The increasing incidence of vancomycin resistance in enterococci [VRE] is necessary to report because of its epidemiological impact in hospital settings where it has been related with outbreaks in many 
centers in several countries. ${ }^{4,5}$ An increasing prevalence of high level aminoglycoside resistance [HLAR] among clinical isolates of enterococci has been reported ${ }^{1}$ and a combined resistance of glycopeptides and high level aminoglycoside are extremely difficult to treat contributing devastating therapeutic consequences.5, 6 The accretion and spread of antibiotic resistance determinants among enterococci, to the point where some clinical isolates are resistant to all standard therapies highlight both the vulnerability of our present armament as well as the looming prospect of a 'post antibiotic era'.3

SUBJECTS AND METHODS: The study was conducted at a tertiary care hospital in Gujarat between April 2010 to June 2011 following an approval from Human Research Ethics Committee.

The study included all the patients presenting to our hospital from which enterococci were isolated from various clinical specimens submitted to Microbiology Laboratory for culture and sensitivity testing. Various clinical specimens were received and accepted at Microbiology laboratory according to acceptance and rejection criteria. The specimens were inoculated in appropriate culture media and the organism was identified at species level using mini API system. The antimicrobial sensitivity was also performed using mini API system whereas minimum inhibitory concentration (MIC) of Vancomycin and High Level Gentamicin were determined using Epsilometer test (E-test) by disk diffusion method. The reporting was done as per Clinical Laboratory Standards Institute (CLSI) guidelines.

Details of patients positive for enterococcal isolates were collected. Data was entered on the same day of the data collection using Microsoft Excel 2007 and a master chart was prepared. Observational tables, charts, and graphs were made as per the data derived. Comparison of results obtained in present study was made with results of previous studies using chi square test and statistical significance was noted.

RESULTS: During the study period 62 Enterococcus species were isolated which accounted for 3\% of total isolates grown in Microbiology laboratory. The distribution of species is shown in Table 1.

Majority of isolates were from extreme age group patients where $23(32 \%)$ isolates were from patients having age of more than 60 years followed by ten (12\%) isolates from children less than 10 years old. The number of male patients 36 (58\%) was more as compared to female patients $22(35 \%)$. With respect to distribution of enterococcal species in wards and intensive care units, 41 $(66 \%)$ isolates were from wards, 17 (27\%) were from intensive care units whereas four (7\%) isolates were from outpatients. Urine was the most common clinical specimen from which enterococci was isolated accounting for $58 \%$ followed by blood and pus each accounting for $16 \%$ of all clinical specimens.

\begin{tabular}{|l|c|}
\hline \multicolumn{1}{|c|}{ Enterococcal species } & Number (\%) \\
\hline E.faecalis & $25(40.32)$ \\
\hline E.faecium & $20(32.25)$ \\
\hline E.gallinarum & $11(17.74)$ \\
\hline E.casseliflavus & $1(1.61)$ \\
\hline E.avium & $1(1.61)$ \\
\hline
\end{tabular}

Table 1: Species distribution of Enterococci $(n=62)$ 
Forty four (71\%) of all enterococcal isolates were found to be resistant to high level aminoglycoside where 37 (59\%) of enterococcal isolates showed combined resistance to high level gentamicin and high level streptomycin. MIC of all isolates that showed high level gentamicin resistance was more than $1024 \mu \mathrm{g} / \mathrm{ml}$ by E-test.

Eight (13\%) enterococcal isolates were resistant to vancomycin from which five were E.faecium and three were E.gallinarum. None of E.faecalis isolates showed resistance to vancomycin. The MIC of VRE ranged from 32 to $>256 \mu \mathrm{g} / \mathrm{ml}$ by E test method. Six VRE strains were isolated from urine whereas two were isolated from blood. Seven out of the eight VRE isolates were nosocomially acquired whereas one was acquired from community.

In present study, ampicillin and penicillin resistance among all enterococci were $58.06 \%$ and $61.29 \%$ respectively. E.gallinarum showed highest resistance to cell wall active agents. It was 100\% and $91 \%$ for penicillin and ampicillin respectively.

DISCUSSION: Enterococci, though commensal in adult faces are important nosocomial pathogen. ${ }^{7}$ Their emergence in past two decade is in many respects attributable to their resistant to many commonly used antimicrobial agents (aminoglycosides, cephalosporins, aztreonam, semi synthetic penicillins, trimethoprim- sulphamethoxazole). ${ }^{8}$

Until recently, Enterococcus faecalis had been the predominant enterococcal species, accounting for 80 to $90 \%$ of all clinical isolates, and Enterococcus faecium had accounted for 5 to 15\%. 5, 7 Other Enterococcus species (E. gallinarum, E. casseliflavus, E. durans, E. avium, and E. raffinose) were isolated much less frequently and accounted for less than $5 \%$ of clinical isolates. 9, 10 In the present study although E.faecalis $(n=25,40 \%)$ and E.faecium $(n=20,32 \%)$ were the predominant enterococcal species E.faecalis constituted for $55 \%$ of both isolates indicating that incidence of E.faecium was high in present study. Besides this a higher incidence of E.gallinarum $(\mathrm{n}=11,18 \%)$ in present study suggests that E.faecium and E.gallinarum are emerging enterococcal species in our institution.

In present study with respect to different clinical specimen positive for enterococci 36 (58.06\%) isolates were from urine samples, ten (16.12\%) were each from blood and pus samples and two $(3.44 \%)$ were from body fluids. Previous studies ${ }^{4,11}$ have shown urinary tract as most common site of infection constituting for $67-82 \%$ followed by soft tissue infections (13 $15 \%)$,blood streaminfections (1 - 18\%) and body fluid (1-3\%).

Previous studies have regarded enterococci predominantly as a nosocomial pathogen in past several years 1,3,5,7 whereas the present study showed that 31 (50\%) isolates were community acquired. This observation is of concern and demands further studies to confirm the findings so as to know actual prevalence and susceptibility pattern of enterococcal isolates in community.

In the present study, out of 62 enterococcal isolates, 38 (61\%) isolates were found to be resistant to Penicillin and 36 (59\%) isolates were resistant to Ampicillin. Previous studies have shown resistance to Penicillin ranging from 1-29\% in enterococcal isolates ${ }^{4,11,12}$ whereas resistance to Ampicillin ranging from $0-67 \%$ in various enterococcal isolates. $2,4,11,12$ Resistance to betalactams was found to be significantly high $(\mathrm{P}<0.05)$ in the present study.

An interesting difference was noted when beta-lactams resistance was compared between hospitals acquired and community acquired enterococcal strains. Twenty six (84\%) of hospital 
acquired strains were resistant to beta-lactams (Penicillin and/or Ampicillin) compared to only 12 (39\%) community acquired strains. This difference was statistically significant $(P=0.001)$ suggesting that prevalence of beta-lactams resistance is more common in hospital acquired strains and this could be a reason why hospital acquired enterococcal infections are difficult to treat.

In present study, eight (13\%) enterococcal isolates were resistant to vancomycin where five isolates were E.faecium and three isolates were E.gallinarum. Previous studies have demonstrated 0 - 6\% resistance to Vancomycin irrespective of enterococcal species. ${ }^{4,6,11,12,13}$

With respect to Vancomycin resistant strains, five (63\%) isolates were E.faecium and three (37\%) isolates were E.gallinarum. There was no vancomycin resistance detected in E.faecalis. The MIC of vancomycin in vancomycin resistant E.faecium ranged from $32->256 \mu \mathrm{g} / \mathrm{ml}$ in present study that was higher when compared to previous studies where it ranged from $8-32 \mu \mathrm{g} / \mathrm{ml}$. [11,13] In addition, all the three vancomycin resistant strains of E.gallinarum had an MIC of $>256 \mu \mathrm{g} / \mathrm{ml}$. Previous study have shown that E.gallinarum strains possessing Van A gene have high level vancomycin resistance and concomitant resistance to teicoplanin. ${ }^{14}$ As incidence of E.gallinarum is high in present study, resistance to vancomycin with such high MIC is a matter of concern as it could have a significant impact on patient's recovery.

Epidemiological distribution of vancomycin resistant strains demonstrated that six out of eight of these isolates were hospital acquired whereas two of them were community acquired. Previous study has also shown incidence of community acquired VRE. 13,15

In present study high level gentamicin and/or streptomycin resistance was detected $(n=44$, 71\%). In previous studies 4, 6,11,12 have demonstrated 13 - 46\% high level aminoglycoside resistance irrespective to enterococcal species. This resistance was more in case of E.faecium (90\%) compared to E.faecalis (72\%). Though resistance of high level aminoglycoside in E.faecium did not have any difference from previous studies 4, 6, 11,12,16 HLAR in E.faecalis was observed to have a significant difference $(\mathrm{P}=0.009)$ compared to previous studies. Combined resistance of high level gentamicin and high level streptomycin in both E.faecalis and E.faecium when taken together was found to have significant difference $(\mathrm{P}=0.000)$ compared to a previous study. ${ }^{4}$ Among the urinary isolates high level aminoglycoside resistance was found to be significantly higher in present study $(\mathrm{P}=0.000)$ compared to a previous study. ${ }^{4}$

With respect to epidemiological types, 28 (90\%) of hospital acquired strains showed HLAR whereas $16(52 \%)$ of community acquired strains showed HLAR. This difference was significant $(\mathrm{P}=0.002)$ suggesting that HLAR was more prevalent amongst the hospital acquired strains probably because of various risk factors as demonstrated in previous studies. ${ }^{12,17}$ When this difference was applied at species level, eight isolates of community acquired E.faecalis showed HLAR where all 10 isolates of hospital acquired E.faecalis showed HLAR. This difference was statistically significant $(\mathrm{P}=0.03)$. For E.faecium and E.gallinarum prevalence of HLAR amongst hospital acquired and community acquired did not show any significant difference.

The MIC or high level gentamicin amongst the HLAR resistant strains was consistently found to be $>1024 \mu \mathrm{g} / \mathrm{ml}$ irrespective to various species suggesting that $100 \%$ of these strains showed a very high level resistance.

To conclude, Enterococci are identified as major pathogens causing infections in hospital as well as community. A high degree of antimicrobial resistance in present study is alarming and poses therapeutic dilemmas for clinicians. Formulating an antimicrobial policy and its strict 
implementation and regular surveillance of enterococcal infections and their antimicrobial profile should be conducted and timely and appropriate infection control measures should be initiated to control their spread.

\section{REFERENCES:}

1. Sanchez ML, Barrett MS, Jones RN. Use of the E Test to predict high - level resistance to aminoglycosides among enterococci. J Clin Microbiol 1992; 30: 3030-2.

2. Schulz JE, Sahm DF. Reliability of the E Test for Detection of Ampicillin, Vancomycin and High level Aminoglycoside Resistance in enterococci spp. J Clin Microbiol 1993; 31: 3336-9.

3. Huycke MM, Sahm DF, Gilmore MS. Multiple - Drug resistant Enterococci: The Nature of the Problem and Agenda for the future. Emerging Infectious Diseases 1998; 4: 239-49.

4. Galindo J, Tejada Y, Cerezo S, Salazar O, Reyes E. High-level aminoglycoside resistance enterococci spp. in a tertiary care hospital in Mexico. Electron J Biomed. 2005; 1: 40-5.

5. Marothi YA, Agnihotri H, Dubey D. Enterococcal resistance -- an overview. Indian J Med Microbiol 2005; 23: 214-9.

6. Bingen E, Doit C, Lambert-Zechovsky N, Tod M, Petitjean O, Bourgeois F, Mariani-Kurkdjian P. Bactericidal activity of daptomycin against vancomycin-resistant Enterococcus faecium in an in vitro pharmacokinetic model. Eur J Clin Microbiol Infect Dis 1991; 10: 1062-5.

7. Tripathi K. Beta Lactam Antibiotics. Chapter 49. Essential of Medical Pharmacology. Fifth Edition. Rep. 2003: 653-67.

8. Iaria C., Stassi G., Costa GB, Di Leo R., Toscano A., Cascio A. Enterococcal meningitis caused by Enterococcus casseliflavus. First case report. BMC Infect. Dis 2005; 5: 2334-5.

9. Bach PB, Malak SF, Jurcic J, et al. Impact of infection by Vancomycin resistant Enterococcus on survival and resource utilization for patients with leukemia. Infect Control Hosp Epidemiol 2002; 23: 471-4.

10. Still J, Law E, Friedman B, Fuhrman S, Newton T. Vancomycin resistant organisms on a burn unit. South Med J 2001; 94:810-2.

11. Luna Adhikari. High-level aminoglycoside resistance and reduced susceptibility to vancomycin in nosocomial enterococci. Journal of Global Infectious Disease 2010; 2: 231-5.

12. Moaddab SR, Rafi A. Prevalence of vancomycin and high level aminoglycosides resistant among High - risk patients. Southeast Asian J Trop Med Public Health 2003; 34: 849-54.

13. Taneja N, Rani P, Emmanuel R, Sharma M. Significance of vancomycin resistant enterococci from urinary specimens at a tertiary care centre in northern India. Indian J Med Res 2004:119:72-4.

14. Facklam RR. Recognition of Group D Streptococcal species of Human Origin by Biochemical and Physiological test. Applied Microbiology 1972; 23:1131-9.

15. Sahm D, Boonlayangoor S, Schulz J. Detection of High - level Aminoglycoside Resistance in enterococci other than Enterococcus faecalis. J Clin Microb 1991; 29: 2595-8.

16. Sanchez ML, Barrett MS, Jones RN. Use of E test to predict high level aminoglycosides resistance among enterococci. J Clin Microbiol 1992; 30:3030-2.

17. Axelrod P, Talbot GH. Risk factor for acquisition of gentamicin - resistant enterococci, a multivariate analysis. Arch Intern Med 1989; 149:1397-401. 
Acknowledgement: None.

\section{AUTHORS:}

1. Amar Pandya

2. M.B. Shah

3. Sunil Trivedi

4. Suman Singh

\section{PARTICULARS OF CONTRIBUTORS:}

1. Microbiologist, Department of Microbiology, H.M. Patel Center for Research and Education, Anand Dist.,

2. Ex Professor and Head, Department of Microbiology, H.M. Patel Center for Research and Education, Anand Dist.,

3. Professor, Department of Microbiology, H.M. Patel Center for Research and Education, Anand Dist.,
4. Professor, Department of Microbiology, H.M. Patel Center for Research and Education, Anand Dist.,

\section{NAME ADDRESS EMAIL ID OF THE} CORRESPONDING AUTHOR:

Dr.Amar Pandya,

3, Saivilla bun,

Nr. Palika Nagar, Bakroal Road,

Anand - 388001.

Email-akupandya@gmail.com

Date of Submission: 14/12/2013.

Date of Peer Review: 16/12/2013.

Date of Acceptance: 25/12/2013.

Date of Publishing: 08/01/2014 